\title{
Rheology and tribology: investigating the migration of small molecules in recycled agricultural waste multilayer films
}

\author{
Géraldine Cabrera, Gérard Pichon, Abderrahim Maazouz and Khalid Lamnawar
}

Géraldine Cabrera. Université de Lyon, CNRS, UMR 5223, Ingénierie des Matériaux Polymères, INSA Lyon, F-69621 Villeurbanne, France.

Gérard Pichon. Groupe BARBIER, Sainte-Sigolène, France.

Abderrahim Maazouz. Université de Lyon, CNRS, UMR 5223, Ingénierie des Matériaux Polymères, INSA Lyon, F-69621

Villeurbanne, France.

Khalid Lamnawar. Université de Lyon, CNRS, UMR 5223, Ingénierie des Matériaux Polymères, INSA Lyon, F-69621 Villeurbanne, France.

Corresponding author: khalid.lamnawar@insa-lyon.fr

\begin{abstract}
This paper deals with polyisobutylene (PIB) migration through post-consumer agricultural waste multilayer films based on four linear low-density polyethylene (LLDPE) matrices. Connections between shear, elongational rheology and tack surface properties were revealed for both model and recycled blends. The effects of aging time and temperature were investigated and rationalized, depending on the short- and long-chain branching in LLDPE matrices as well their polyethylene (PE) crystallization. Linear and nonlinear viscoelastic properties were influenced by even small amounts of PIB. This migration also influenced slippage in the steady-flow regime. Transient uniaxial extensional properties were shown to be very sensitive to the presence of PIB, which seems to hold back the PE strain hardening properties. Therefore, the axial force and the surface friction coefficient were determined and discussed in correlation with bulk rheological findings. These results help unveil new insights about the physical mechanisms governing PIB migration with or without fillers inhibiting this migration in recycled films.
\end{abstract}

Keywords. Rheology, Multilayers, Recycling, Polymer Blend, Microstructure, Polymer Melt, Extensional Flow

\section{Introduction}

The use of flexible multilayer films has become essential in the agricultural sector, allowing a remarkable increase in agricultural production, earlier harvesting and reduced plagues [1]. For such applications, the most common polymer used is linear low-density polyethylene (LLDPE), followed by low-density polyethylene (LDPE), high-density polyethylene (HDPE) and ethylene-vinyl acetate (EVA) [2]. Most consumers, however, have a negative perception of plastic packaging due to the considerable amount of waste it produces in their daily lives. In the European Union, about 25 million tons of post-consumer plastic waste is generated every year. The total volume of flexible consumer packaging was predicted to increase from 27.4 million tons (2017) to 33.5 million tons (2020) [2]. In a circular economy context, with the dual problems of depletion of natural resources and the environmental impact of a growing volume of waste, it is absolutely essential to focus on the recycling process of multilayered plastic films.

This study focuses on the valorization of post-consumer agricultural waste films, specifically bale wrapping films. The waste films coming from bale wrapping films go through an open-loop recycling process that involves waste collection, recycling and reuse of the multilayer waste films as a secondary raw material for new applications. The mechanical recycling process used takes the multilayered films through different stages such as washing, shredding, drying and pelletizing.

The basic requirements of bale wrapping films consist on the multilayer film ability to properly stretch around the desire product to be enfolded and the residual tension necessary to give it a tight contour wrap [3]. In order to obtain a film with autohesive surface, a polyisobutylene (PIB) is added into a polyethylene matrix. The PIB is a pressure-sensitive 
Rheology and tribology: investigating the migration of small molecules in recycled agri...

adhesive (PSA) and the most predominant cling agent used on stretch-wrapping films. Polyisobutylenes can range from waxy solids to extremely viscous liquids depending on their molar mass. Rennert et al. [8] have demonstrated that two phases coexist in PE/PIB blends and the PIB molecules distribute themselves into the amorphous phase.

The literature to date indicates that the main factors influencing polyisobutylene (PIB) migration kinetics through a polyethylene matrix are the co-monomer type, density, crystallinity, molecular structure and the molar mass of the matrix used, as well as the PIB molar mass. In 2014, Rennert et al. used a mechanical cling test and atomic force microscopy (AFM) to investigate the cling and adhesion forces. They found that cling forces increase with aging time, reaching a maximum two weeks after preparation. Also, they observed that high molar mass PIB diffuses more slowly through an LLDPE matrix compared to a PIB of lower molar mass. Moreover, it was observed that the storage conditions of the films have a direct impact on the cling properties and need to be considered for further investigations.

In the context of the valorization of post-consumer agricultural waste films, it has been a particular phenomenon was observed during the manufacturing process, in which recycled waste films were reused as raw material at the inner layer. As explained before, when the bale wrapping films are first used, PIB provides the surface of the films with cling properties. Nonetheless, after the recycling process, this PIB migration represents a drawback for the industrial process and limits the reuse of the waste films as a raw material. This phenomenon has created an enticement to investigate and understand the mechanisms that govern the PIB diffusion process in the aim of finding possible solutions. Therefore, the main objective of this study is to develop a deep understanding of PIB migration in post-consumer agricultural waste films [4].

To attain these objectives, model LLDPE/PIB blends of different compositions were prepared before transposing the approach to real-life recycled materials, which are complex, multiphase systems. We sought to investigate the effect of PIB diffusion and its dependence on the nature and temperature of the PE matrix. This involved an original approach making use of new techniques such as the tack test and tribo-rheometry.

\section{Experimental section}

\subsection{Materials for the preparation of model blends}

The materials used in this study are shown in Table 1 and Table 2. The four different LLDPE matrices were selected to simulate the different constituents of their equivalent multilayer structure. Model blends based on these four LLDPE matrices and PIB were prepared as shown in Table 3.These blends are rich in LLDPE, since it is the most usual polymer found in bale wrapping waste films. Then, in order to facilitate the preparation of the reference films, an LFigure 11Figure 11LDPE/PIB masterbatch was used. Furthermore, mineral fillers (CaCO3 and talc) were added to the LLDPE/ PIB blends. To prevent dispersion issues, we also worked with masterbatches.

Table 1 Summary of polyethylene matrices selected

\begin{tabular}{|c|c|c|c|c|}
\hline Polymer & LLDPE1 & LLDPE2 & LLDPE3 & LLDPE4 \\
\hline Mw (g/mol) & $93747^{a}$ & $113251^{a}$ & $108780^{a}$ & $115506^{\mathrm{a}}$ \\
\hline Density $\left(\mathrm{g} / \mathrm{cm}^{3}\right)$ & 0.918 & 0.918 & 0.919 & 0.923 \\
\hline $\begin{array}{l}\text { MFI }\left(190^{\circ} \mathrm{C} / 2.16 \mathrm{~kg}\right) \\
(\mathrm{g} / 10 \mathrm{~min})\end{array}$ & 1 & 0.9 & 0.9 & 0.2 \\
\hline
\end{tabular}


Table 2 Summary of additives and mineral fillers used

for this study

\begin{tabular}{|c|c|c|c|c|}
\hline Material & PIBh300 & MPIB & MTalc & $\mathrm{MCaCO}_{3}$ \\
\hline Masterbatch & - & $\begin{array}{c}70 \pm 2 \% \text { PIBh300 } \\
\text { in LLDPE }\end{array}$ & $\begin{array}{c}75 \pm 2 \% \text { talc in } \\
\text { LLDPE }\end{array}$ & $\begin{array}{c}80 \pm 2 \mathrm{CaCO}_{3} \\
\text { in LLDPE }\end{array}$ \\
\hline $\mathrm{Mw}(\mathrm{g} / \mathrm{mol})$ & $1300^{\mathrm{a}}$ & - & - & - \\
\hline Density $\left(\mathrm{g} / \mathrm{cm}^{3}\right)$ & - & 0.91 & 1.85 & 2.02 \\
\hline \multicolumn{5}{|r|}{} \\
\hline \multicolumn{7}{|r|}{} \\
\hline
\end{tabular}

Monolayer films were obtained by blown extrusion using an annular blow die of $50 \mathrm{~mm}$ diameter coupled to the melt pump to prepare the LLDPE/PIB (Table 3). The temperature profile during extrusion was gradually increased from $190^{\circ} \mathrm{C}$ to $220^{\circ} \mathrm{C}$ from feeding to compression to the pumping zone. Next, the molten polymer in the form of a tube exited from the die at $220^{\circ} \mathrm{C}$ and then was drawn upward by a take-up device. For the present study, a drawing speed of $3.7 \mathrm{~m} / \mathrm{min}$ was used in order to obtain films with similar thicknesses of around $30 \pm 5 \mu \mathrm{m}$. All films were prepared with the same extrusion parameters, including the recycled blends (Table 4).

Finally, to validate the study with the model blends, recycled blends were studied also. As shown in Table 4 and based on the source of the waste films, two types of recycled multiphase systems were selected. The objective was to compare PIB migration in films subjected to different aging times and storage temperature conditions after the manufacturing process. A mechanical recycling process was employed, taking the multilayered waste through various stages such as washing, shredding, drying and pelletizing. Finally, a blown extrusion process was applied to the resulting pellets in order to produce monolayer films.

Table 3 Model film compositions with different LLDPE matrices

\begin{tabular}{|c|c|c|c|c|c|c|}
\hline $\begin{array}{l}\text { LLDPE1 (wt } \\
\%)\end{array}$ & $\begin{array}{l}\text { LLDPE } 2 \\
\text { (wt \%) }\end{array}$ & $\begin{array}{l}\text { LLDPE } 3 \\
\text { (wt \%) }\end{array}$ & $\begin{array}{l}\text { LLDPE } 4 \\
\text { (wt \%) }\end{array}$ & $\begin{array}{l}\text { PIBh300 } \\
\text { (wt \%) }\end{array}$ & $\begin{array}{l}\text { Talc } \\
\text { (wt \%) }\end{array}$ & $\begin{array}{l}\mathrm{CaCO}_{3} \\
\text { (wt \%) }\end{array}$ \\
\hline 95 & - & - & - & 5 & - & - \\
\hline- & 95 & - & - & 5 & - & - \\
\hline - & - & 95 & - & 5 & - & - \\
\hline- & - & - & 95 & 5 & - & - \\
\hline 85 & - & - & - & 5 & 10 & - \\
\hline 85 & - & - & - & 5 & - & 10 \\
\hline
\end{tabular}

Table 4 Recycled multiphase blends originating from waste collection

\begin{tabular}{ccc} 
Material & Principal composition & Waste source \\
\hline $\mathrm{R} 1$ & LLDPE/LDPE/PB & Post-consumer \\
\hline $\mathrm{R} 2$ & LLDPE/LDPE/PIB & Post-production \\
\hline $\mathrm{R} 1+10 \% \mathrm{CaCO}_{3}$ & LLDPELDPE/PIB/CaCO & Post-consumer with $\mathrm{CaCO}$ \\
\hline
\end{tabular}

\subsection{Probe tack test: Experiments with a rotational rheometer}

Tack is the resistance offered by an adhesive film upon its detachment from a substrate, i.e., the adhesive ability to 
Rheology and tribology: investigating the migration of small molecules in recycled agri...

form an instant bond when brought rapidly into low-pressure contact with a solid surface [5] .The probe tack test, also known as the "quick stick" test, is one of the many techniques available to measure the tack of pressure-sensitive adhesives (PSAs) [6].

In previous studies, McNally, Small, et al. and Rennert et al. measured the tack level of polyethylene films containing PIB using the peel test with a tensile test machine. In the present work, we performed our tack measurements following the ASTM D279-01 procedure, but using a DHR-2 rotational rheometer with parallel-plate geometry instead, which makes our protocol unique. Because in our case the tack test was used primarily to investigate the migration of PIB into the surface of the films, the DHR-2 was more suitable in terms of axial force sensitivity, with a range of $0.1 \mathrm{~N}$, to measure the maximum force required to separate the probe from the sample. In our study, a 40-mm steel parallel-plate geometry was selected, because a high debonding resistance is favored by a large contact area [5]. The films were fixed onto the lower plate using double-sided bonding tape. The tack experiments were then performed at room temperature $\left(25^{\circ} \mathrm{C}\right)$. The experimental protocol consists of two steps: (i) compression between the upper plate and the film with an axial force of $5 \mathrm{~N}$, and (ii) after 1 second of contact the upper plate is detached from the film at a constant rate of $0.1 \mathrm{~mm} / \mathrm{s}$.

In order to obtain reproducible results, the upper plate was cleaned with acetone after each experiment [9]. More than 30 experiments were performed at different locations of the films. The maximal axial force was then directly obtained, along with its standard deviation.

In order to evaluate the evolution of the tack as a function of storage temperature conditions, the samples were conditioned prior to analysis in an air-circulating oven at $40^{\circ} \mathrm{C}$ and at room temperature for up to 30 days [4].

\subsection{Tribo-rheometry: Tack measured as a coefficient of friction}

We considered that the resistance to the rolling motion of a ball on a PSA reflects the tackiness of the adhesive, since the motion is closely related to the bonding and debonding processes, which appear simultaneously at the contact surface. It is possible to express the tack in terms of a rolling coefficient of friction that depends on the physical properties of the materials [5].

The objective was to use this measurement technique to quantify the migration of PIB through the polyethylene film surface, thereby providing complementary information to the earlier study involving tack measurements. Using a triborheometry accessory installed on the DHR-2 rheometer, it is possible to measure the dynamic friction coefficient as a function of frequency and temperature. The tribo-rheometry geometry chosen is called "three balls on a plate." This geometry consists of three spheres arranged on a circle of $15 \mathrm{~mm}$ radius and a lower plate with the substrate attached. The spheres have a diameter of $7.94 \mathrm{~mm}$ and provide a point contact with the substrate. As for the tack test, the films were fixed onto the lower plate using a double-sided bonding tape. All tribo-rheometry experiments were performed at room temperature using the following protocol: apply a fixed axial force of $2.5 \mathrm{~N}$, which is the force required for sufficient contact with the sample without wrinkling it, and measure the evolution of the load and friction force as a function of the angular velocity over a range from $1.10-4 \mathrm{rad} / \mathrm{s}$ to $15 \mathrm{rad} / \mathrm{s}$.

In tribo-rheometry, speed-dependent changes in the frictional properties are portrayed through Stribeck curves, in which the dynamic friction coefficient $(\mu)$ is plotted as a function of the sliding speed. The equations that define the variables in this study are as follows: 


$$
\begin{gathered}
F_{f}=M / d \\
v_{s}=d \times \omega \\
\mu=M / d F_{N}
\end{gathered}
$$

where Equation (1) describes the frictional force " $F f$ " with "M" as the torque [N.m] and "d" as the arm [m]; Equation (2) the sliding speed with " $\omega$ " as the rotational angular speed [rad/s] and Equation (3) the dynamic friction coefficient " $\mu$ " with $\mathrm{F}_{\mathrm{N}}$ as the axial force [N]. With increasing speed, the PIB at the surface of the film is transported into the space between the film and the three balls that are in contact with it. For this reason, hydrodynamic friction is the most suitable regime for evaluating and quantifying PIB migration through the surface of the film. As with the probe tack test, the evolution of the tack was considered as a function of storage temperature conditions. The samples were conditioned prior to analysis in an air circulating oven at $40^{\circ} \mathrm{C}$ and at room temperature for up to 30 days.

\section{Probing of PIB migration: Quantitative study}

\subsection{Tack test: Effect of the nature of the PE matrix on PIB migration}

The architecture of the polyethylene matrix is crucial for understanding the diffusion mechanism of the PIB. We selected four different LLDPEs with diverse chemical structures, crystallization morphologies and rheological behavior in the molten state. The results displayed in Figure 1 and Table 5 show that as of day 7 of aging time, it is possible to observe differences in PIB diffusion through the four LLDPEs. The two extremes are the LLDPE1 blend, which reached 3.4 $\mathrm{N}$ of average axial force, compared with the LLDPE4 blend, with only $0.5 \mathrm{~N}$ at day 7. For the LLDPE2 and LLDPE3 blends, diffusion is also slower than for LLDPE1. 

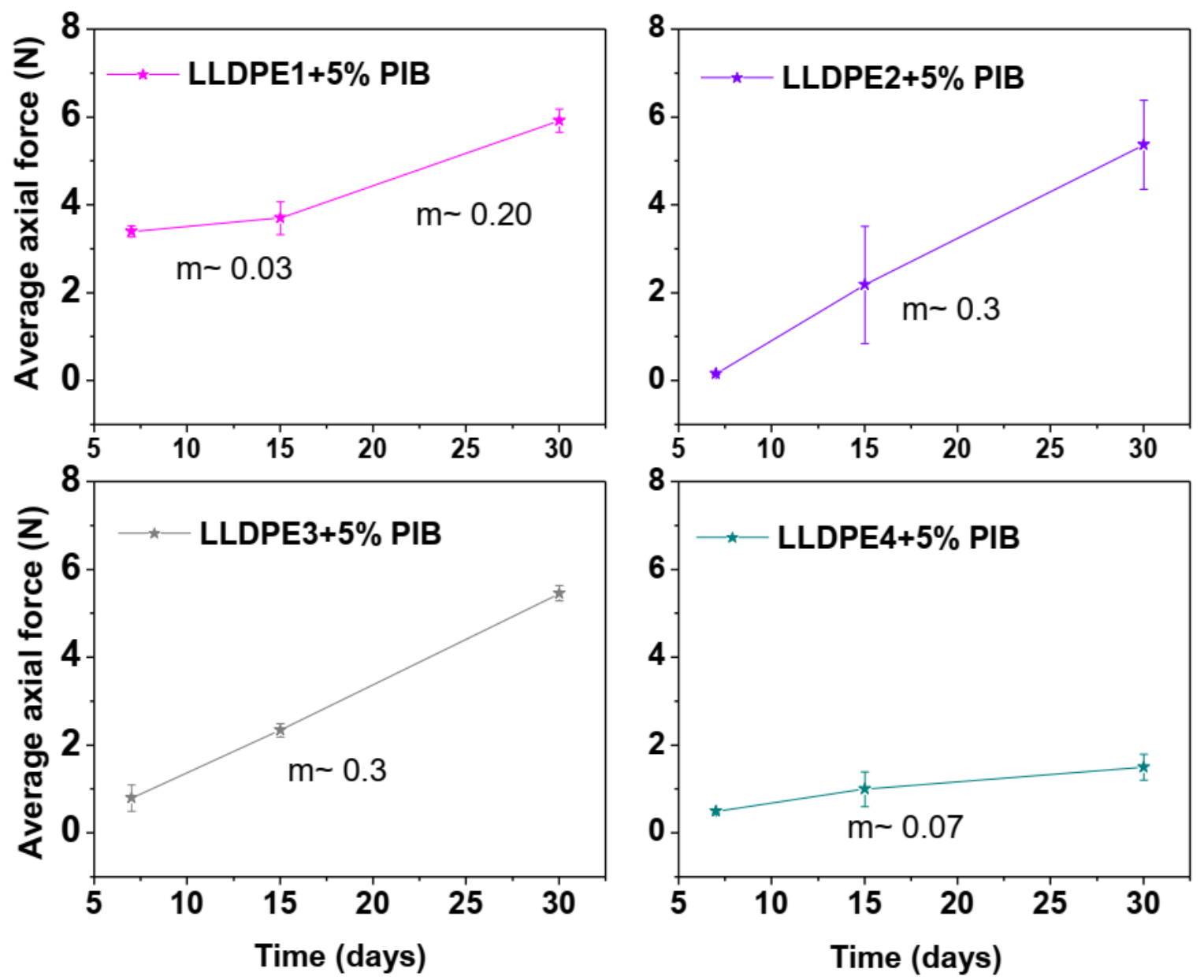

Figure 1. Influence of the nature of the PE matrix on the migration of PIB. Evolution of the average axial force as a function of aging time, with an aging temperature of $25^{\circ} \mathrm{C}$ and “ $\mathrm{m}$ " representing the slope of the migration kinetics as

$$
\sim(\mathrm{Dt})^{\mathrm{m}} \text {. }
$$

It appears that LLDPE1 is the matrix that most easily allows PIB molecules to migrate to the surface. On the other hand, the LLDPE4 blend is the complete opposite of LLDPE1, as indicated in Table 5, which shows that the average axial force at day 30 of aging reached only $1.5 \mathrm{~N}$ for LLDPE4. It is important to mention that the PIB diffusion kinetics is the same for all of the polyethylene matrices, since the average axial force increases with aging time. For example, at day 30 of aging, the LLDPE1, LLDPE2 and LLDPE3 blends reached nearly the same value of axial force, but the standard deviation of LLDPE2 was much higher than that of the other two, reflecting the heterogeneity of PIB diffusion through the LLDPE2 matrix. Then, considering the Axial Force as the PIB concentration at the surface of the films and in function of time, we calculate the slope of each LLDPE axial force plotted in Figure 1 in order to check if the migration process was neo-Fickian or not. As observed, the LLDPE2/PIB and LLDPE3/PIB blends present values closer to the theoretical 0.5 slop acting that the PIB migration could be correlated to the measured force and its linked to ( Dt)m, where D is the PIB diffusion coefficient and " $m$ " is the slope. In case of LLDPE1/PIB, the slope from 7 to 15 days is slower than the one of 15 to 30 days, which indicates that the migration is accelerated from day 15 of aging time and could be close to a neo-Fickian process. Finally, the LLDPE4/PIB system presents the slowest slope "m", which reflects a non-neo-Fickian PIB migration. These results can be related to the different microstructure of each LLDPE, which affect the PIB diffusion process [4].

Table 5 Summary of the average axial force as a function of aging time and temperature 


\begin{tabular}{l|l|l|l}
\hline LLDPE1+ 5\%PIB & $3.4 \pm 0.1$ & $3.7 \pm 0.4$ & $5.9 \pm 0.3$ \\
LLDPE2+ 5\%PIB & $0.2 \pm 0.1$ & $2.2 \pm 1.3$ & $5.4 \pm 1.0$ \\
LLDPE3+ 5\%PIB & $0.8 \pm 0.3$ & $2.3 \pm 0.2$ & $5.5 \pm 0.2$ \\
LLDPE4+ 5\%PIB & $0.5 \pm 0.0$ & $1.0 \pm 0.4$ & $1.5 \pm 0.3$ \\
\hline
\end{tabular}

\begin{tabular}{c|c|c|c}
\hline \multirow{2}{*}{ Film } & $\begin{array}{c}\text { Average axial force } \\
\text { (N) }\end{array}$ & $\begin{array}{c}\text { Average axial force } \\
\text { Day } 7\end{array}$ & $\begin{array}{c}\text { Average axial force (N) } \\
\text { Day 30 }\end{array}$ \\
\hline
\end{tabular}

Aging temperature @ $25^{\circ} \mathrm{C}$

\subsection{Tribo-rheometry: Effect of the nature of the PE matrix on PIB migration}

Tribo-rheometry was selected in order to quantify PIB migration in the films, under the widest possible range of industrial conditions. For the tribo-rheometry curves, the dynamic friction coefficient $(\mu)$ was plotted as a function of the Gumbel number. The Gumbel number (Gu), described in Equation (4), is widely used in tribo-rheometry to consider the influence of the lubricant viscosity at the sample surface, the axial force and the sliding speed applied during the test. In our study, the PIB plays the role of lubricant in tribo-rheometrical terms. Hence, using Equation (4), the Gumbel number [10] was calculated using 64.5 Pa.s as the PIB viscosity at room temperature, where " $\mathrm{R}$ " is the radius of the sphere in contact with the sample, " $V_{\mathrm{s}}$ " the sliding speed, " $\mathrm{d}$ " the arm and " $\mathrm{F}_{\mathrm{n}}$ " the axial force applied.

$$
G u=\frac{\eta\left(\pi R^{2}\right) V_{S}}{d\left(F_{n}\right)}
$$

Before comparing the two techniques to evaluate the effect of the mineral fillers, we must specify that it is impossible to directly compare axial force values with the dynamic coefficient of friction, since they are completely different parameters and were not obtained under the same conditions. The intention here is to place both techniques side by side and observe if the results tend to agree.

The experiments presented in this section were performed on the films on aging day number 30 and at room temperature $\left(25^{\circ} \mathrm{C}\right)$. LLDPE1 was the matrix chosen to study the influence of mineral fillers, since this film with PIB is the stickiest at the surface. Starting with the tack test results shown in Figure 12b, there is a huge gap between the average axial force of the LLDPE1 blend with PIB and that of the films containing mineral fillers. LLDPE1+5\%PIB presents an average axial force of $5.9 \mathrm{~N}$, while films with $\mathrm{CaCO}_{3}$ and talc reach only 0.4 and $0.2 \mathrm{~N}$, respectively (Table 6).

As for the tribology results shown in Figure 2a, we normalized the friction coefficient of the films with PIB and mineral fillers, in order to evaluate only the effect of PIB at the surface and neglect the friction coefficient that talc and $\mathrm{CaCO}_{3}$ present by themselves. This is described in Equation (5), where " $\varepsilon$ PIB" represents the coefficient that takes into account only the PIB at the surface of the films containing mineral fillers. In Figure 2a is represented a Stribeck curve, as it is 
Rheology and tribology: investigating the migration of small molecules in recycled agri...

called in tribology. While the sliding speed increases, the PIB at the surface of the films is transported into the space between the film and the balls which are in contact, since the upward forces of the PIB push the surfaces apart (mixed friction). Then, when the sliding speed is further increased, the PIB at the surface gets thicker. The internal friction of the PIB then increases, and consequently the friction of the entire tribo-system is also augmented [4]. This regime at high sliding speed is called Hydrodynamic friction. Since the objective is to evaluate the "Tackiness" of the films in terms of a rolling coefficient of friction, we considered only the final part of the Stribeck curves, which corresponds to the hydrodynamic friction. This section represents the bonding and debonding process between the rolling balls and the film, and it simulates the widest range of friction encountered in industrial conditions [4].

Then, we can observe the same tendency as with the tack test, where LLDPE1 with PIB and mineral fillers exhibits a decrease of the system friction coefficient.

$$
\varepsilon_{P I B}=\frac{\mu_{P E+P I B+\text { fillers }}}{\mu_{P E+\text { fillers }}}
$$



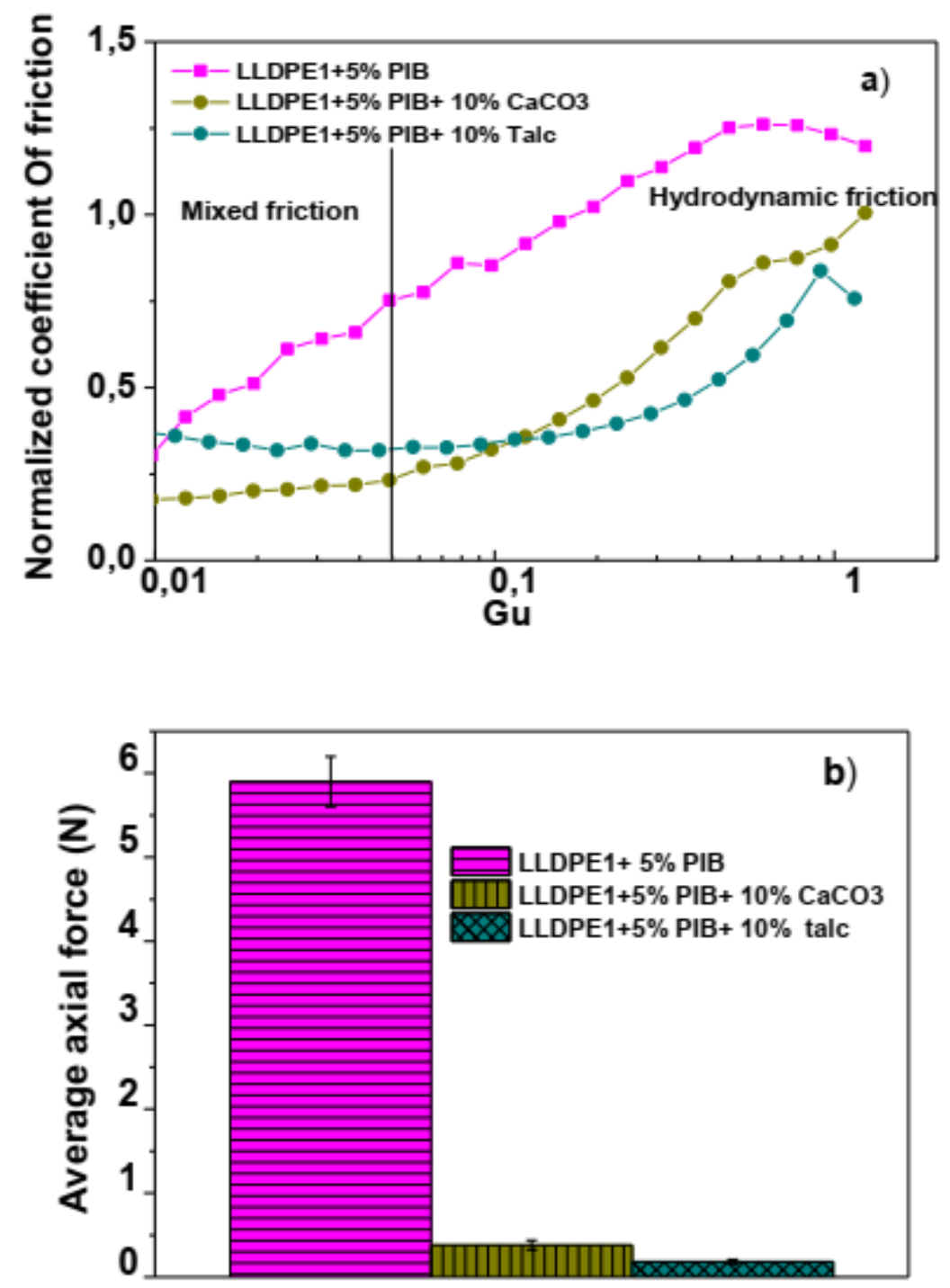

Figure 2. LLDPE1 $+5 \%$ PIB and influence of mineral fillers at $25^{\circ} \mathrm{C}$. a) Evolution of the kinetic coefficient of friction, normalized by the friction of each mineral filler, as a function of the Gumbel number. b) Histogram of average axial

forces.

These results clearly indicate that mineral fillers are able to decrease the tack of the films with PIB. The particles are dispersed in the amorphous phase of the LLDPE matrix and by consequence act as a migration barrier, preventing the PIB from passing through it [8]. Talc seems to be the most effective mineral filler when it comes to blocking the diffusion of the PIB molecules. Even if $\mathrm{CaCO}_{3}$ and talc have the same particle size $(2 \mu \mathrm{m})$, it is the shape that matters. The $\mathrm{CaCO}_{3}$ particles are spherical, while the talc particles are laminar. These laminar-shaped particles dispersed through the amorphous phase of polyethylene present a more difficult obstacle to the migration of the PIB molecules.

\subsection{Tack test vs. tribo-rheometry: Validation with recycled films}

So far we have focused on model monolayer films rich in LLDPE, in order to better understand the migration mechanism of PIB. Nevertheless, as we recalled previously, PIB migration limits the reuse of waste films as a raw material in an 
Rheology and tribology: investigating the migration of small molecules in recycled agri...

industrial context. Therefore, since the two techniques of characterization proposed in this article have been validated with the model films, the studies were transposed to the recycled blends.

Table 6 Summary of the average axial force as a function of the aging time and temperature for LLDPE1-matrix films and recycled films.

\begin{tabular}{c|c}
\hline Film & $\begin{array}{c}\text { Average axial force (N) } \\
\text { Day 30 }\end{array}$ \\
\hline \multicolumn{2}{c}{ Aging temperature @ 25 ${ }^{\circ} \mathrm{C}$} \\
\hline LLDPE $1+5 \%$ PIB & $5.9 \pm 0.3$ \\
LLDPE $1+5 \%$ PIB $+10 \% \mathrm{CaCO}_{3}$ & $0.4 \pm 0.1$ \\
LLDPE $1+5 \% \mathrm{PIB}+10 \%$ talc & $0.2 \pm 0.0$ \\
\hline $\mathrm{R} 1$ & $1.1 \pm 0.4$ \\
$\mathrm{R} 2$ & $0.5 \pm 0.1$ \\
$\mathrm{R} 1+10 \% \mathrm{CaCO}_{3}$ & $0.1 \pm 0.0$
\end{tabular}

The recycled blends emanated from post-consumption and post-production waste. All of the waste films were recognized as bale wrapping films as their first use. Both of the recycled blends were identified as having an approximate composition of 70\% LLDPE, 12\% LDPE and 5\% PIB. However, even though the two blends share the same composition, they experienced different lifetimes. Before the recycling process, R1 had already experienced its first use-life, meaning exposure to various temperature conditions, UV radiation, humidity, etc. Meanwhile, R2 came directly from production waste, but had also experienced long storage time and varying temperatures.

As shown in Figure 3b and Table 6, the high standard deviation of R1 indicates that its PIB migration was less homogeneous than with R2. Even though the two recycled blends have the same composition, it is very possible that they are not made with the same LLDPE matrix. Then, considering the tribology results in Figure 3a, the evolution of the kinetic coefficient of friction of R1 also indicates heterogeneous PIB migration at the surface of the films, since at some points of the hydrodynamic friction section, the R2 shows a higher coefficient of friction than R1.

Finally, we can observe that mineral fillers act as a migration barrier for the recycled blends also. As shown in Figure $3 \mathrm{~b}$ and Table 6 , the presence of $10 \% \mathrm{CaCO}_{3}$ decreased by a factor of 10 the average axial force measured by the tack test. As for the tribology results in Figure 3b, the kinetic friction coefficient of R1 decreased from 1.15 to 0.85 . 

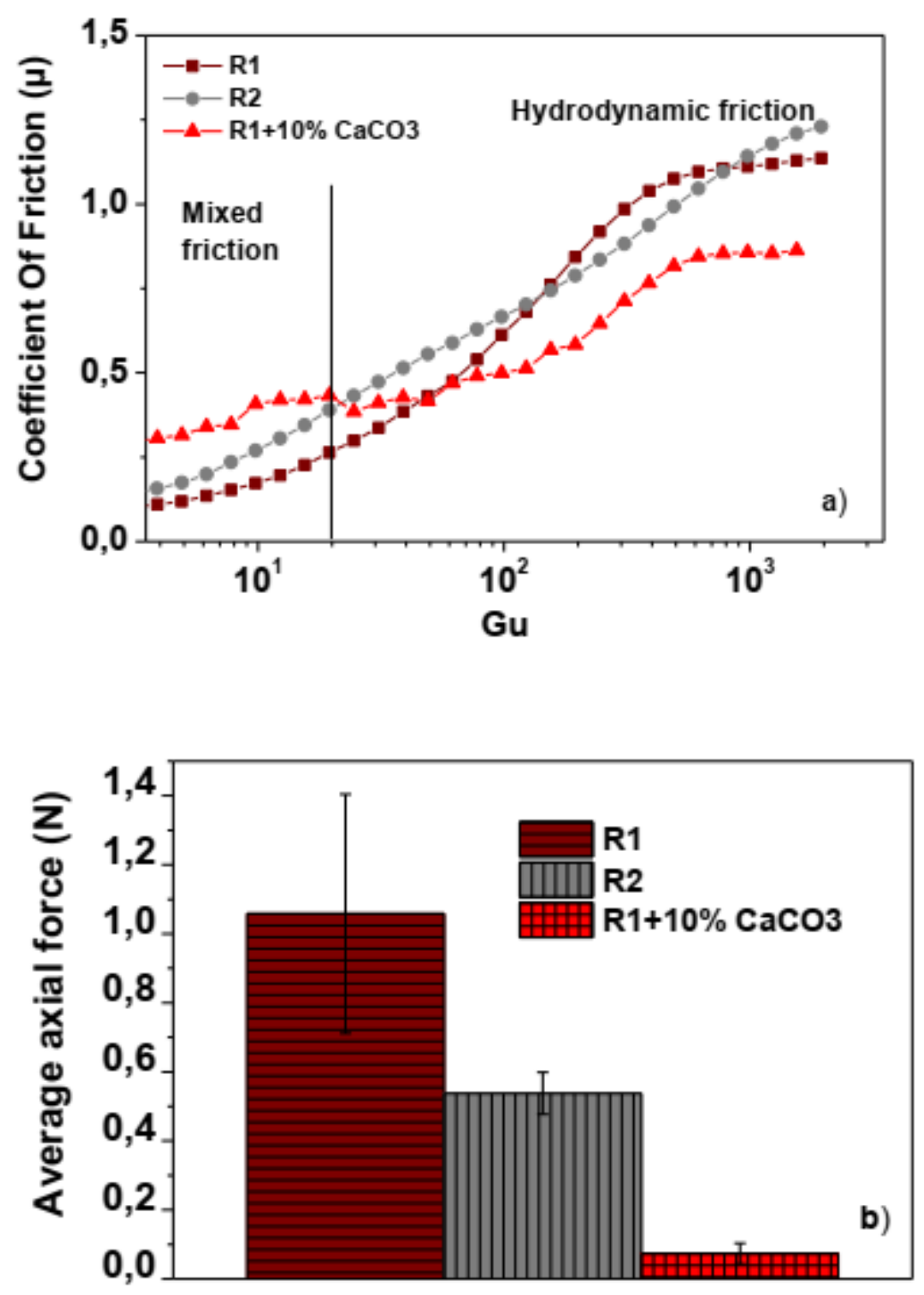

Figure 3 Recycled films and influence of mineral fillers at $25^{\circ} \mathrm{C}$. a) Evolution of the kinetic coefficient of friction, normalized by the friction of each mineral filler, as a function of the Gumbel number. b) Histogram of average axial force.

These results demonstrate the efficacy of the tack test and tribology for quantifying PIB migration, even with blends as complex as the recycled blends [8]. Naturally, the recycled blends exhibited a lower level of tack in terms of axial force, since these blends contain approximately 12\% LDPE. The presence of LDPE can influence PIB migration through the LLDPE+LDPE matrix blend. Our results also show that mineral fillers can decrease the tack of films containing PIB, including recycled blends.

\section{Conclusions}

The migration phenomenon PIB on the post-consumer agricultural waste films became our motivation to investigate and understand the mechanisms that govern the Polyisobutylene (PIB) diffusion. The PIB is as pressure sensitive adhesive (PSA) added to the primitive multilayer systems in order to obtain an autohesive surface for bale wrapping films applications. However, this PIB migration to the surface is not required in the recycled multiphase system. 
Rheology and tribology: investigating the migration of small molecules in recycled agri...

Since the recycled materials are complex blends, model systems based on different polyethylene matrices and PIB were studied. Four different LLDPE matrices were selected, with the objective to investigate the influence of their molecular architecture on the PIB migration. A quantitative study was performed and specifically developed, using Tack test and Tribo-rheometry to quantify and model the PIB diffusion process depending of aging time and temperature. Moreover, after obtaining coherent results between Tack test and Tribo-rheometry, we selected Talc and $\mathrm{CaCO}_{3}$ as mineral fillers to add into the blends of LLDPE and PIB. These fillers act as a migration barrier, blocking the PIB molecules to pass through it. Then, Talc seems to be the most effective to block the PIB molecules to diffuse, since laminar shaped particles dispersed through the amorphous phase of polyethylene present a more difficult obstacle to the PIB molecules to migrate, thanks to their higher specific surface.

Finally, transposing the study into the characterization of recycled blends, same tendency of results were obtained with both characterization techniques. These demonstrate the efficacy of Tack test and Tribo-rheometry to quantify the PIB migration, even with blends as complex as the recycled blends. With the help of these techniques, we were able to prove that mineral fillers are able to decrease the tack of the films with PIB, including the recycled blends. Mineral fillers are an excellent solution to the industrial development of blown multilayer films, using waste wrapping films as a raw material.

\section{Acknowledgements}

This research project was financially supported by the Auvergne-Rhône-Alpes Regional Council (ARC AURA program 2017-2020) and the Groupe Barbier.

\section{Bibliography}

[1] CEPLA and P. Europe, Plastics in agriculture-applications and usages handbook, J.C Lopez,. Almeria, 2009.

[2] O. Horodytska, F. J. Valdés, and A. Fullana, "Plastic flexible films waste management - A state of art review," Waste Management, vol. 77, pp. 413-425, 2015.

[3] G. M. McNally, C. M. Small, W. R. Murphy, and A. H. Clarke, "The effect of PIB molecular weight on the cling characteristics of polyethylene-PIB films for stretch and cling film applications," Journal of Plastic Film and Sheeting, vol. 21, no. 1, pp. 55-68, 2005.

[4] G. Cabrera, J. Charbonnier, G. Pichon, A. Maazouz, and K. Lamnawar, "Bulk rheology and surface tribo-rheometry toward the investigation of polyisobutylene migration in model and recycled multilayer agricultural films," Rheologica Acta, vol. 59, no. 11, pp. 821-847, 2020.

[5] I. Benedek, Pressure-Sensitive Adhesives and Applications. Second Edition, Revised and Expanded, 2nd, illustr ed. New York.Basel, 2004.

[6] C. L. Rohn, “Analysis of tack,” MRS Bulletin, vol. 28, no. June, pp. 434-439, 2003.

[7] G. M. McNally, C. M. Small, W. R. Murphy, and G. Garrett, "The effect of vinyl acetate content and polyisobutylene concentration on the properties of metallocene polyethylene-ethyl vinyl acetate coextruded film for stretch and cling film applications," Journal of Plastic Film and Sheeting, vol. 21, no. 1, pp. 69-83, 2005.

[8] M. Rennert et al., "Investigation of the migration behavior of polyisobutylene with various molecular weights in ethylene/ $\alpha$-olefin copolymer blown stretch films for improved cling properties," Journal of Applied Polymer Science, vol. 131, no. 10, pp. 1-13, 2014. 
[9] B. E. Gdalin, E. V. Bermesheva, G. A. Shandryuk, and M. M. Feldstein, "Effect of temperature on probe tack adhesion: Extension of the dahlquist criterion of tack," Journal of Adhesion, vol. 87, no. 2, pp. 111-138, 2011.

[10] H. P. Kavehpour and G. H. McKinley, "Tribo-rheometry: From gap-dependent rheology to tribology," Tribology Letters, vol. 17, no. 2, pp. 327-335, 2004.

PDF automatically generated on 2021-05-25 10:19:25

Article url: https://popups.uliege.be/esaform21/index.php?id=737

published by ULiège Library in Open Access under the terms and conditions of the CC-BY License (https://creativecommons.org/licenses/by/4.0) 